\title{
Clustered DNA damage induced by heavy ion particles
}

\author{
Hiroaki Terato and Hiroshi Ide
}

Department of Mathematical and Life Sciences, Graduate School of Science, Hiroshima University, 1-3-1 Kagamiyama, Higashi-Hiroshima 739-8526, Japan

\begin{abstract}
Clustered DNA damage (locally multiply damaged site) is thought to be a critical lesion caused by ionizing radiation, and high LET radiation such as heavy ion particles is believed to produce high yields of such damage. Since heavy ion particles are major components of ionizing radiation in a space environment, it is important to clarify the chemical nature and biological consequences of clustered DNA damage and its relationship to the health effects of exposure to high LET particles in humans. The concept of clustered DNA damage emerged around 1980, but only recently has become the subject of experimental studies. In this article, we review methods used to detect clustered DNA damage, and the current status of our understanding of the chemical nature and repair of clustered DNA damage.
\end{abstract}

\section{Introduction}

Among all the biomolecules which make up a living organism, it is widely accepted that deoxyribonucleic acid (DNA), which carries the cell's vital genetic information, is the primary target of ionizing radiation (IR). The acceptance of the fact that DNA is the target of IR has been guided by much direct and indirect evidence derived from the result of extensive research in radiation biology conducted over a long period of time. Considering the spatial structure of energy transfer resulting from exposure to IR, discrete DNA lesions can be generated on DNA strands along the track of IR (i.e. isolated damage). In addition, multiple lesions can be produced on DNA strands when a single track of ionizing radiation hits the DNA directly or passes close by the DNA strand. This type of lesion is referred as clustered DNA damage, or as a locally multiply damaged site (LMDS) (Ward, 1981).

There is a spectrum of radiation present in space environments. Considering the effect of radiation on human health in space, two types of radiation are important: solar radiation and galactic cosmic rays. The former consists primarily of protons, and the latter contains various types of high energy heavy ion particles (HZE particles) (Simpson, 1983). HZE particles are the major source of radiation exposure to humans in space (Hagen, 1989). In addition, HZE particles have high linear energy transfer (LET) values and deposit a large amount of energy along their tracks, with the potential to produce increased amounts of clustered DNA damage. Therefore, it is important to understand how clustered

Review

Received: January 4, 2005

Address for correspondence: Dr. Hiroaki Terato Department of Mathematical and Life Sciences, Graduate School of Science, Hiroshima University, 1-3-1 Kagamiyama, Higashi-Hiroshima 739-8526, Japan E-mail; hterato@hiroshima-u.ac.jp
DNA damage is produced by HZE particles and how this damage is processed in cells. Reviews are available covering this subject (Ward, 1988, 1994; Goodhead, 1994), and the aim of this review is to focus on the methods used to detect clustered DNA damage, and on current understanding of the chemical nature and repair of clustered DNA damage.

\section{Nature of clustered DNA damage}

Exposure to IR can result in the deposition of energy to DNA molecules leading to DNA damage. Since IR traverses cells as a beam or as a flow of particles, DNA damage arises along the track of the IR. Thus, unlike most genotoxic chemicals, DNA damage induced by IR is localized. The level of localized DNA damage is believed to increase with increasing LET values of the radiation. Because LET is an index or measure of the energy transferred to an object along the path of the radiation, high LET radiation can deposit more energy than low LET radiation (ICRU report, 1970). Condensed or concentrated energy deposition results in clusters of ionization events such as spurs, short tracks and branch tracks. Consequently, such ionization clusters can yield numerous lesions in a small volume containing the target molecule, leading to augmentation of the resulting damage. When the target is DNA, the site of such lesions is termed clustered DNA damage or LMDS. Since the diameter and the length of a helical turn of naked double stranded DNA are 2-3 nm, even the smallest unit of ionization clusters (a spur) can span a region of a DNA helix. Clustered DNA damage can also occur when the track of the IR travels sufficiently close to a DNA molecule. Although a rigorous definition of clustered DNA damage is not found in the literature, this type of damage is considered to have a latent capacity which results in a double strand break (DSB) in a DNA molecule. We usually consider damage to be clustered DNA damage when the site of the damage contains multiple (two or more) lesions in a portion of the DNA which encompasses just a few helical DNA turns. 
To characterize clustered DNA damage, it is necessary to consider the type, number, and the spatial distribution of the DNA lesions. The type of DNA lesions induced by IR are the same regardless of whether they are inside or outside of the clustered DNA damage region, and includes base lesions, sugar lesions, strand breaks, and cross-links (von Sonntag, 1987). Most IR-induced DNA lesions are generated by reactive oxygen species (ROS) derived from the ionization of water which is present in abundance in living cells. Consequently, this type of DNA damage is similar to endogenous damage induced by ROS generated as by-products of normal aerobic metabolism (Fig. 1). The number of lesions present in a region of clustered DNA damage may vary depending on the nature of the radiation and is a topic of recent studies (see below). The spatial distribution of lesions in clustered DNA damage is also important. Lesions can be present on one strand or distributed on the two complementary strands of DNA. In addition, the distance between lesions on opposing strands is crucial, since two closely spaced lesions on opposing strands could lead directly to the formation of a DSB, or indirectly to a DSB as the consequence of the action of DNA repair enzymes. Two closely spaced single-strand breaks (SSBs) on complementary strands will result in a DSB. A DSB is one of the forms of clustered DNA damage and was the only detectable consequence of clustered DNA damage in the earlier history of radiation biology. Although most researchers realized that a DSB is a type of damage characteristic of IR, the concept of clustered DNA damage did not emerge for a long time. The experimental analysis of clustered DNA damage containing base lesions was made possible by the use of specific DNA repair enzymes that incise DNA at base lesions (Wallace, 1998). Recent progress in the procedures used to detect clustered DNA damage is described below.

\section{Theoretical studies and physical considerations of clustered DNA damage}

The concept of clustered DNA damage developed from theoretical studies of the track structure of IR. Ionization events induced by IR are rare and stochastic

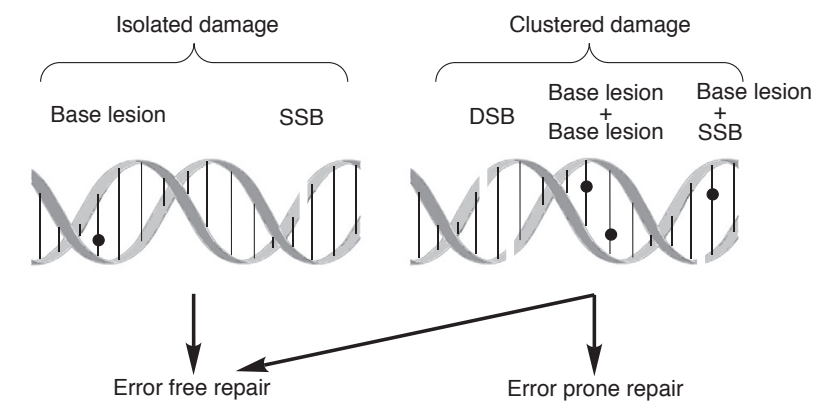

Fig. 1. Types of isolated and clustered DNA damage induced by ionizing radiation. events, and are modeled in theoretical studies working with computer simulations of actual events. The form and pattern of energy deposition is important in causing the loss of activity in a target molecule. First, the radius of the penumbra describing the energy deposition pattern resulting from the passage of a single IR particle was thought to be important in determining the cross section of a target molecule; this concept was introduced by Butts and Katz in 1967. The scattering angle and energy of released electrons produced along a track of IR were used to estimate the spatial pattern and pathway of the electrons. It was shown that at a microstructure level, the delivered dose decreases with an increase of distance from the IR track center. More recently, simulations were used to predict the route of each electron derived from a single IR track. Monte Carlo track simulations were derived using experimentally determined target cross sections. A number of track simulations or codes were developed for photons, electrons and heavy ion particles. The comparison of the results of these track simulations or behavior codes is summarized in Nikjoo et al. (1998), and Michalik (1992) found a good agreement between the yields of DSBs obtained using theoretical and experimental protocols. The latter data were obtained from in vivo experiments using classical methods such as neutral filter elution assays (see below). According to this study, the yield of isolated damage events decreases with increases in the LET values, whereas the yield of clustered damage events increases with increases of LET up to $100 \mathrm{keV} / \mu \mathrm{m}$. However, Roots et al. (1990) reported contradictory results showing an inverse correlation of LET and the yield of DSBs in vivo. It was also shown that the energy of the IR is an important factor in determining the yield of DSBs. The yield of DSBs increased with increases of the energy of He ion particles from $33 \mathrm{keV} / \mu \mathrm{m}$ to $130 \mathrm{keV} / \mu \mathrm{m}$.

The lifetime of the hydroxyl radical is $\sim 8.7 \times 10^{-9}$ $\mathrm{s}$, from which that the average diffusion distance was determined to be $9.3 \mathrm{~nm}$ (Roots and Okada, 1975). Since this distance is 3 to 4 times larger than the diameter and the length of a turn of double helical DNA, it is possible that DNA damage induced by an IR track can be dispersed over more than one helical turn. Charge migration in a DNA strand may also lead to alterations or differences between the sites of energy deposition and the sites where the final damage is manifested (Hall et al., 1996). An electron generated by the direct effect of IR in a DNA strand can migrate over $4 \mathrm{~nm}$, which is larger than one helical turn of the DNA, and this occurs within $10^{-1} \mathrm{~s}$ via the $\pi$-stacking of adjacent base pairs. The dose rate is also an important factor in vivo, because the generation and repair of clustered DNA damage are competing processes. The efficiency of recovery from DSBs induced by $\gamma$-rays in human fibroblast HF19 cells decreases with an increase in the dose rate (Foray et al., 1996). The energy of the IR is also important (Michalik, 1992) because high energy IR can produce a larger 
number and more energetic $\delta$-rays. Thus, a number of physical factors affect the formation of clustered DNA damage.

\section{In vitro studies of clustered DNA damage}

\section{Supercoiled plasmid DNA}

Many in vitro studies have been performed to provide simple and accessible models for understanding complicated events induced by IR in vivo. DSBs, which were the early research focus of clustered DNA damage research, can also be generated by physical shearing during experimental handling. Therefore, small DNA molecules such as plasmid and phage DNA molecules have been used for this type of experiment since they are more resistant to shearing during handling. DSBs were most extensively examined during the early stages of these studies, because there was no detection methods for other types of clustered DNA damage, e.g. such as base damage clusters. One of the useful model systems used for DSB analysis is the detection of conformational changes in supercoiled plasmid DNA. When the supercoiled form (type I) of intact plasmid DNA is inflicted with a single strand break (SSB), it unwinds and changes from type I form to a nicked open-circular form (type II) plasmid. The occurrence of a single DSB in type I DNA leads to its assuming a linear form (type III). The three different forms of plasmid (type I, type II, and type III) can be separated using appropriate procedures. Initially, studies based on this principle were reported by Christensen et al. (1972) for the comparison of DSBs and SSBs induced by heavy ion particles. Different forms of $\phi X 174$ DNA (replicative form, 5,386 bp) were separated by alkaline sucrose density gradient centrifugation. According to this study, the yield of SSBs (representing isolated damage events) decreased linearly with the increase of LET values, but that of DSBs (representing clustered DNA damage events) increased with LET values. Later, Van Touw et al. (1985) performed a similar study using neutral agarose gel electrophoresis for the separation and analysis of irradiated plasmid DNA. Gels were stained to visualize the DNA, and the images of the DNA bands were analyzed using image analysis software (e.g. NIH image) (Fig. 2). Recently, this model system has been applied to the detection of base damage. This protocol and its use for analysis is made possible owing to the availability of copious recombinant repair enzymes involved in base excision repair (BER), which are primarily DNA glycosylases. Escherichica coli formamidopyrimidine-DNA glycosylase (Fpg) excises oxidative purine lesions such as 8-oxoguanine and formamidopyrimidine from the DNA backbone. E. coli thymine glycol-DNA glycosylase (endonuclease (Endo) III) excises oxidative pyrimidine lesions such as thymine glycol and urea. Fpg and Endo III are bifunctional DNA glycosylases containing an additional AP lyase activity, which incises an AP site and generates a strand break.
Thus, the treatment of plasmid DNA containing base damage with these enzymes results in conformational changes of the plasmid DNA, and these are detectable using agarose gel electrophoresis. Fpg and Endo III have been widely used for this purpose.

Jones et al. (1993) compared the yield of damage induced by $\gamma$-rays and ${ }^{4} \mathrm{He}$ particles in SV40 DNA $(5,243$ bp) under radical scavenging conditions (TE buffer containing $1 \mathrm{mM}$ dimethylsulfoxide (DMSO)). They showed that the numbers of SSBs induced by $\gamma$-rays and ${ }^{4} \mathrm{He}$ were 3.25 and 0.39 sites/Mbp/Gy, respectively. The numbers of DSBs induced by $\gamma$-rays and ${ }^{4} \mathrm{He}$ were 0.052 and 0.036 sites/Mbp/Gy, respectively. Thus the yields of DSBs after exposure to $\gamma$-rays and ${ }^{4} \mathrm{He}$ are 63 and 11 times lower than those of SSBs, respectively. Other work also reported that the yield of clustered DNA damage events is lower than that of isolated damage events by a factor of 10 to 100 . Using pUC18 (2,686 bp), Milligan et al. (1996) compared the yields of SSBs and DSBs induced by $\gamma$-rays and ${ }^{4} \mathrm{He}$ particles in phosphate buffer

A

Agarose gel electrophoresis (plasmid DNA)

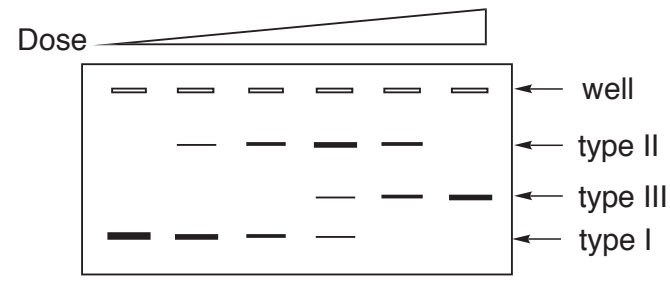

Plasmid DNA conformations

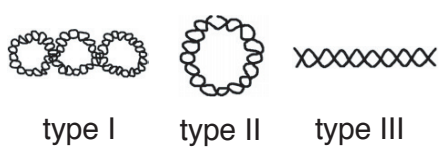

B

\section{Dose response curves of types I - III}

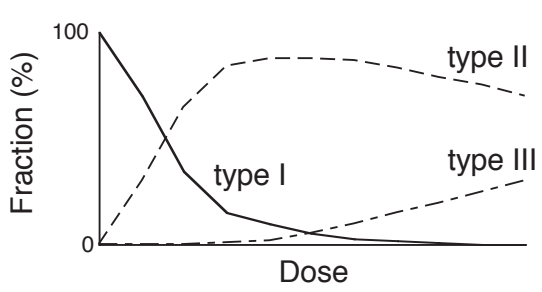

Fig. 2. In vitro assay of clustered DNA damage using supercoiled plasmid DNA. (A) Diagram of agarose gel electrophoresis of plasmid DNA molecules with different conformations generated by ionizing radiation. (B) Dose response curves for three types of plasmid DNA. 
containing $10 \mathrm{mM}$ DMSO. According to this report, $\gamma$-rays and ${ }^{4} \mathrm{He}$ produced 1.74 and $0.31 \mathrm{SSBs} / \mathrm{Mbp} / \mathrm{Gy}$, respectively. DSBs induced by $\gamma$-rays and ${ }^{4} \mathrm{He}$ were 0.006 and 0.009 sites/Mbp/Gy, respectively. Taucher-Sholz et al. (1999) performed a systematic study with various types of IR. They irradiated SV40 DNA in TE buffer with $\mathrm{X}$-rays and HZE particles including ${ }^{4} \mathrm{He},{ }^{12} \mathrm{C},{ }^{16} \mathrm{O},{ }^{20} \mathrm{Ne}$, ${ }^{40} \mathrm{Ar},{ }^{41} \mathrm{Ca},{ }^{48} \mathrm{Ti},{ }^{56} \mathrm{Fe},{ }^{59} \mathrm{Ni},{ }^{84} \mathrm{Kr},{ }^{131} \mathrm{Xe},{ }^{197} \mathrm{Au},{ }^{207} \mathrm{~Pb}$ and ${ }^{228} \mathrm{U}$. They reported that the yield of SSBs is constant at 1.5 sites/Mbp/Gy up to $13 \mathrm{keV} / \mu \mathrm{m}$ and then decreases to 0.46 sites/Mbp/Gy with further increases of LET values up to $200 \mathrm{keV} / \mu \mathrm{m}$. The yield of DSBs is also constant at 0.052 sites $/ \mathrm{Mbp} / \mathrm{Gy}$ up to $13 \mathrm{keV} / \mu \mathrm{m}$, and then decreases to 0.042 sites $/ \mathrm{Mbp} / \mathrm{Gy}$ with increases of LET values up to $200 \mathrm{keV} / \mu \mathrm{m}$. Prise et al. (1999) compared damage in pMSG-CAT DNA $(8,405$ bp) irradiated with $\gamma$-rays and $\alpha$-particles. In TE buffer, the yield of SSBs with $\gamma$-rays and $\alpha$-particles were 0.71 and 0.20 sites/Mbp/Gy, respectively. The frequency of DSBs induced by $\gamma$-rays and $\alpha$-particles was 0.015 and 0.025 sites/Mbp/Gy, respectively. They also estimated the extent of base damage recognized by Endo III (i.e. oxidative pyrimidine lesions). Isolated pyrimidine lesions generated by $\gamma$-rays and $\alpha$-particles were detected at a frequency of 1.89 and 0.16 sites/Mbp/Gy, respectively. Clustered pyrimidine lesions generated by $\gamma$-rays and $\alpha$-particles were detected at a frequency of 0.048 and 0.015 sites/Mbp/Gy, respectively. These results suggest that $\gamma$-rays produce more clustered base damage than DSBs, but $\alpha$-particles show an opposite trend. These results might have been affected by the incision efficiency of Endo III for lesions in clustered DNA damage (see below).

There appears to be a pattern for the generation of damage by HZE particles. The yield of isolated damage decreases with an increase in LET values, whereas clustered DNA damage shows no apparent dependence on LET values. Some studies reported an increase in the yield of clustered DNA damage and others reported a decrease. In all cases, the reported changes were small. These results do not conform with the idea that clustered DNA damage is a key factor for the biological effects of IR because relative biological effect (RBE) values increase with an increase of LET up to 100 or 200 $\mathrm{keV} / \mu \mathrm{m}$. This discrepancy must be examined in future studies.

\section{Linear DNA}

Pulse field gel electrophoresis (PFGE) is used for the separation of linear DNA molecules in the megabasepair size region (Carle and Olsen, 1984; Schwartz and Cantor, 1984). For in vitro model experiments, T7 phage DNA $(39,936 \mathrm{bp})$ and $\lambda$ phage DNA (48,502 bp) have been utilized. One DSB produces two smaller fragments from the original DNA molecule. Thus, the yield of DSBs can be evaluated from the length of the resulting DNA fragments. Irradiated DNA bands produce smears with PFGE, because DSBs occur randomly leading to the formation of a population of DNA molecules with a continuous range of fragment sizes. Therefore, a mathematical treatment is necessary to evaluate the number of DSBs produced in these experiments.

Sutherland's group developed a number-average length analysis for DNA populations to evaluate the numbers of DSBs produced (Fig. 3A) (Freeman et al., 1986; Sutherland et al., 2003). They analyzed clustered DNA damage in T7 DNA using this procedure (Sutherland et al., 2000). In this study, $\gamma$-rays generated 0.14, $0.06,0.16$ and 0.06 sites/Mbp/Gy of DSBs, oxidative pyrimidine clusters, oxidative purine clusters and AP clusters, respectively. The latter three values were obtained by the post-irradiation treatment of irradiated DNA with Endo III, Fpg and Endo IV. Endo IV is an E. coli AP endonuclease which converts an AP site to a strand break. The yield of oxidative pyrimidine clusters obtained with T7 DNA is comparable to that with plasmid systems such as those by Prise et al. (1999), but that of DSBs with T7 DNA is notably higher (ca. 9-fold) than that with plasmid. This difference might be due to the conformational differences of the DNA used and/or the characteristics of the individual detection methods used. Clustered DNA damage containing AP sites is more effectively converted to DSBs by putrescine than by Endo IV (Georgakilas et al., 2002). Similar analysis of clustered DNA damage was extended to a single molecule of DNA using a micro-flow cell with a laser fluorescence detection system (Filippova, et al., 2003). This group also used a modified method for the analysis of clustered DNA damage in chromosomal DNA irradiated with heavy ion particles (Sutherland et al., 1996). Chromosomal DNA was prepared using cell lysis in an agarose plug to avoid the physical shearing of the DNA. The plugs were subsequently treated with the restriction enzyme NotI to obtain measurable sizes of chromosomal DNA (1.2-1.3 Mbp) for a number-average length analysis. The agarose plugs were irradiated with low doses up to $10 \mathrm{cGy}$ and then subjected to PFGE. This study showed that the yield of DSBs was 0.0046 $\mathrm{DSB} / \mathrm{Mbp} / \mathrm{Gy}$ with $\gamma$-rays and that over 10 times more DSBs were generated by Fe particles. The yield of DSBs is significantly lower than that seen in T7 DNA, which may reflect the scavenging environment of chromatin.

\section{In vivo studies of clustered DNA damage}

\section{Classical methods}

Studies of clustered DNA damage in vivo must be designed to manipulate or work with large chromosomal sized DNA. In the early days of radiation biology, various procedures were developed to detect DNA strand breaks induced by IR. Most of these techniques detected DNA strand breaks by looking at decreases in the molecular size of DNA. One of the techniques frequently used for this purpose was neutral density gradient centrifugation. This protocol measured the 
molecular size of DNA by observing its sedimentation in an appropriate density gradient, such as a sucrose density gradient, after ultracentrifugation (McEwen, 1967; Hagen, 1973). Recently, studies using sucrose density gradient centrifugation have become less common, but this method is still useful for determining the molecular mass of a substrate molecule. Neutral filter elution assays derived from the alkaline filter elution technique (Kohn et al., 1976) were used for the evaluation of DSBs. This protocol is based on observations of DNA passing through filters with pores of specific sizes (Bradley and Kohn, 1979; Woods, 1981). The DNA unwinding technique was also utilized for the detection of DSBs (Bryant and Blocher, 1980), and this procedure was originally used for the detection of DNA containing SSBs (Ahnstrom and Erixon, 1973). Double stranded DNA containing SSBs has loose ends leading to partial unwinding at the ends of the breaks. Single and double stranded DNA can also be separated with hydroxyapatite chromatography, and the numbers of strand breaks could be evaluated experimentally (Rydberg, 1975). Detailed descriptions of these classical procedures are reviewed by von Sonntag (1973).

\section{PFGE for in vivo experiments}

In addition to being used for in vitro experiments, PFGE has also been utilized for the detection of DSBs generated in chromosomal DNA after exposure to IR in vivo. However, there are some differences from the in vitro model studies mentioned above. Because the molecular size of mammalian chromosomal DNA is very large, it is easily subjected to physical shearing, and the intact DNA cannot be removed from the sample wells used with the PFGE protocol. Another complication is caused by the fact that chromosomal DNA in mammalian cells is present in a range of sizes. Irradiated cells are embedded in a gel plug and treated with proteinase $\mathrm{K}$ to lyse the cells, and the gel is then subjected to electrophoresis. Most of the chromosomal DNA remains in the well, and only small DNA fragments derived from shearing of the original DNA molecules can exit from the well during electrophoresis. Electrophoresis then generates a DNA band in the gel, and the characteristics of the band correlate with the extent of DNA fragmentation which has occurred. In the fraction of activity released (FAR) assay (Fig. 3B), the amount of DNA exiting from the well is analyzed to estimate the number of DSBs present in the DNA sample. The FAR assay, developed by Blocher (1990) and Blocher and Kunhi (1990), is based on the size of DNA prevented from exiting the well. Since the DNA exiting the well contains only a small fraction of the DNA remaining in the well, the chromosomal DNA in the cells is usually pulse-labeled with $\left[{ }^{3} \mathrm{H}\right]$ thymidine before irradiation to increase assay sensitivity. Alternatively, hybridization was used for DNA detection (Friedl et al., 1993). After electrophoresis, the gel containing the DNA is sectioned and the radioactivity in each fraction is measured. The FAR value is determined using the following equation:

$$
\text { FAR }=\text { c.p.m.(lane)/[c.p.m.(lane) + c.p.m.(well)] }
$$

Finally, the FAR value is converted into the yield of DSBs/cell/Gy using the following equation:

$$
\mathrm{F}_{\text {RETAINED }}=\exp ^{[-\eta \mathrm{D}(\mathrm{k} / \mathrm{m})]}\{1+-\eta \mathrm{D}(\mathrm{k} / \mathrm{m}) \cdot[1-(\mathrm{k} / \mathrm{m})]\}
$$

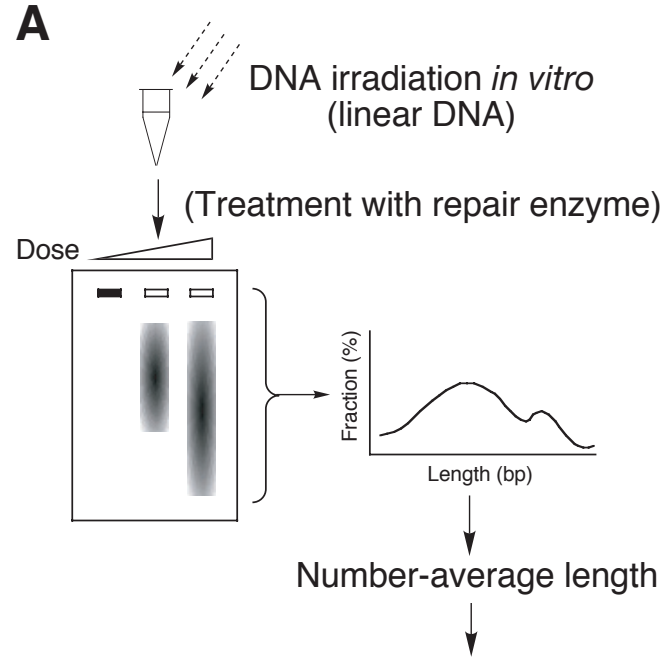

(or Clustered base damage)

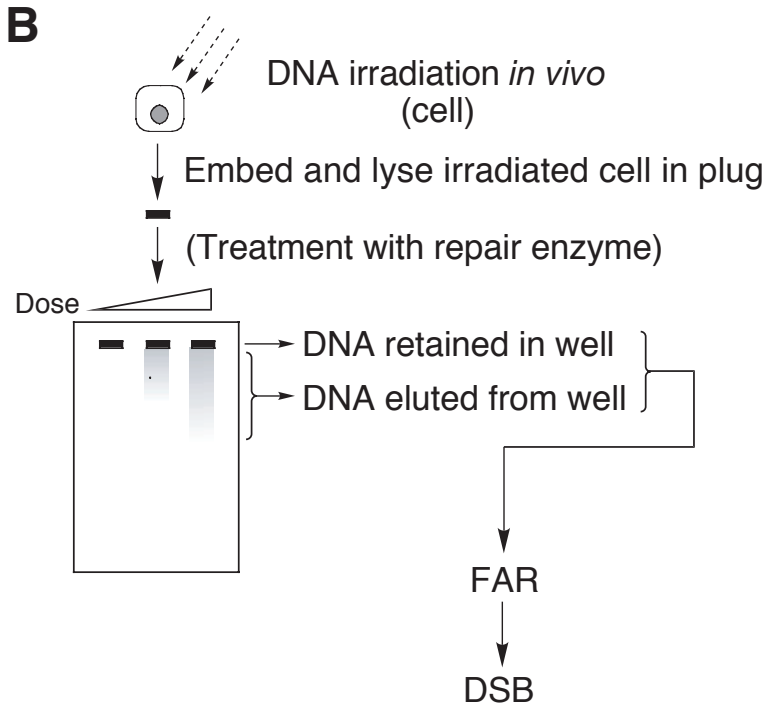

(or Clustered base damage)

Fig. 3. PFGE analysis of clustered DNA damage in linear DNA. (A) Diagram of number-average length analysis using linear DNA (T7 or $\lambda$ DNA) irradiated in vitro. (B) Diagram of the FAR assay for irradiated cells. The yield of DSBs is directly evaluated with these methods, whereas clustered base damage is evaluated by a combination of this method along with treatment of the DNA with repair enzymes. 
where $F_{\text {retained }}$ is 1 - FAR, which is determined with experiment methods, $\eta$ is the mean frequency of DSBs per chromosome per unit of dose, $\mathrm{k}$ is the exclusion size of DNA which is unable to leave the well and progress into the gel, $\mathrm{m}$ is the mean molecular weight of the chromosomes in the irradiated cells, and D is the dose. Gulston et al. (2002) compared the yield of clustered DNA damage using different evaluation systems in vitro and in vivo. In the in vitro system, pUC18 DNA was irradiated in $200 \mathrm{mM}$ Tris to mimic cellular scavenging conditions, and conformation changes were monitored by agarose gel electrophoresis as described above. The in vitro system showed that the yields of DSBs and clustered base damage were 0.01 and 0.017 sites/Mbp/ $\mathrm{Gy}$, respectively. The latter was obtained by simultaneous treatment of the irradiated DNA with Endo III and Fpg. The in vivo system using a FAR assay showed that in vivo DSB and clustered base damage were 0.02 and 0.009 sites/Mbp/Gy, respectively, in human HF19 fibroblast cells, indicating a fairly good agreement with the in vitro data. Weber and Flentje (1993) also showed good agreement in comparing data obtained with filter elution and the FAR assay. As mentioned in the previous section, they reported that the yield of DSBs decreased with an increase in LET values from $\gamma$-rays $(0.23 \mathrm{keV} / \mu \mathrm{m})$ to $\mathrm{Ne}$ ion particles $(450 \mathrm{keV} / \mu \mathrm{m})$, and then increased with heavy ion particles with LET values over $1800 \mathrm{keV} / \mu \mathrm{m}$. However, this result does not conform to the known relationship between LET and RBE. On the other hand, Rydberg (1996) showed that in human skin fibroblast G38 cells, the DSB yield increased with an increase of LET up to $\sim 200 \mathrm{keV} / \mu \mathrm{m}$. Newman et al. (1997) reported on the distinct distributions of DNA fragment sizes generated by $\mathrm{X}$-rays and $\alpha$-particles. The yield of DNA fragments with sizes over $225 \mathrm{kbp}$ is greater after exposure to $\alpha$-particles than to X-rays, suggesting that the loop structure of chromatin is important in the spatial distribution of IR-induced DSBs in vivo. A numberaverage length analysis for clustered DNA damage has also been applied to in vivo studies (Sutherland et al., 2000, 2002).

\section{Comet assay}

The comet assay (single cell gel electrophoresis) has recently become a popular technique for the analysis of clustered DNA damage in vivo (Olive, 1999). This procedure was originally developed for quantifying DNA strand breaks without DNA radiolabeling (Ostling and Johansen, 1984). In this procedure, cells are embedded in an agarose microgel on a slide glass, lysed, and electrophored. This results in elution of fragmented DNA from the nucleus. DNA is stained with an appropriate fluorescent dye, and analyzed by fluorescent microscopy. The DNA eluted from a single cell under these conditions forms a comet shaped band, thus giving the method its name. An advantage of the comet assay is that the degree of DNA damage can be analyzed in individual cells, while the other methods described above produce a damage assessment generated from an entire cell population. The biological effects of IR are stochastic, hence there might be damaged and undamaged cells in the same irradiated population. Jostes et al. (1993) showed that both, hit and intact cells, were present after irradiation with $\alpha$-particles.

However, a problem for the comet assay which has yet to be resolved is how to standardize the results. The shape of the DNA comet electrophoresed from the cells is easily affected by experimental conditions, e.g. electrophoresis conditions, extent of fluorescent staining, capture sensitivity of the gel image, and all of these parameters make it difficult to compare data from individual cells and experiments. Exhaustive attempts were made to standardize the comet assay (Bocker et $a l .$, 1997), but a protocol has not been fully established yet. Many protocols are used to evaluate the comet tails resulting from this method (Bocker et al., 1997). However, the lack of an appropriate standard with cells containing defined amounts of DNA damage makes it difficult to calculate the absolute amount of damage seen with this method, although several groups have made efforts to do this. Olive and Banath (1993) evaluated clustered DNA damage induced by ${ }^{125} \mathrm{I}$-incorporation using the comet assay, and found a good agreement with results obtained using PFGE, and Evans et al. (1995) detected clustered DNA damage containing base lesions using a comet assay combined with DNA glycosylase treatment. The comet assay is also limited with respect to the dose range used, because the comet tail formation shows a saturation effect at higher doses (Olive, 1999), and this could lead to underestimation of the damage present in the cell.

\section{Repair and biological consequences of clustered DNA damage}

The severity of the biological consequences resulting from IR exposure may be related to clustered DNA damage. RBE values for higher organisms such as mammals increase with an increase of LET values up to $\sim 200 \mathrm{keV} / \mu \mathrm{m}$ (ICRU Report 16, 1970). If clustered DNA damage is a key factor involved in the manifestation of the biological consequences resulting from exposure to IR, the cellular processing of clustered DNA damage must be a major concern for radiation biology. Clustered DNA damage exerts genotoxic effects via two mechanisms which are not necessarily mutually exclusive. One is direct genotoxicity, and the other is resistance to DNA repair. DSBs, one consequence of clustered DNA damage, is processed through either nonhomologous end joining (NHEJ) or homologous recombination (HR) (Hoeijmakers, 2001). The latter is an error-free repair pathway and maintains the correct sequence of DNA, because HR uses a homologous sequence that serves as a template for repair. On the other hand, NHEJ usually results in the loss of a few 
nucleotides at the ends of the DNA breaks and results in modifications of any sequence in the damaged region of the DNA, and is an error-prone repair pathway.

It has been shown that the cellular processing of clustered DNA damage containing base lesions could result in enhanced cytotoxic effects (Blocher et al., 1991; Blaisdell and Wallace, 2001). For instance, the attempted repair of clustered DNA damage containing a base lesion and a SSB on two opposing strands could lead to the generation of a DSB which can lead to more severe consequences than the unrepaired damage (Fig. 4). If a base lesion is a component of the clustered DNA damage present, and is removed by bifunctional DNA glycosylases such as hNTH1 and hOGG1 which are human homologues of Endo III and Fpg (Wood et al., 2001), the site of a base lesion will be converted to a strand break by AP lyase activity. Thus, attempted repair can convert this component of clustered DNA damage into a DSB, which is a cytotoxic lesion. Consecutive actions of monofunctional DNA glycosylases and AP endonucleases can produce a similar result. Consistent with this notion, overexpression of such DNA glycosylases in human lymphoblasts increases their sensitivity to IR and for the production of DSBs simultaneously (Yang et al., 2004). A similar result was also obtained by engineering the overexpression of Fpg in E. coli (Blaisdell and Wallace, 2001).

The excision efficiency of DNA glycosylases for a lesion present in clustered DNA damage was first studied by Chaundhry and Weinfeld (1995). Dihydrothymine (DHT) located opposite to a strand containing a SSB

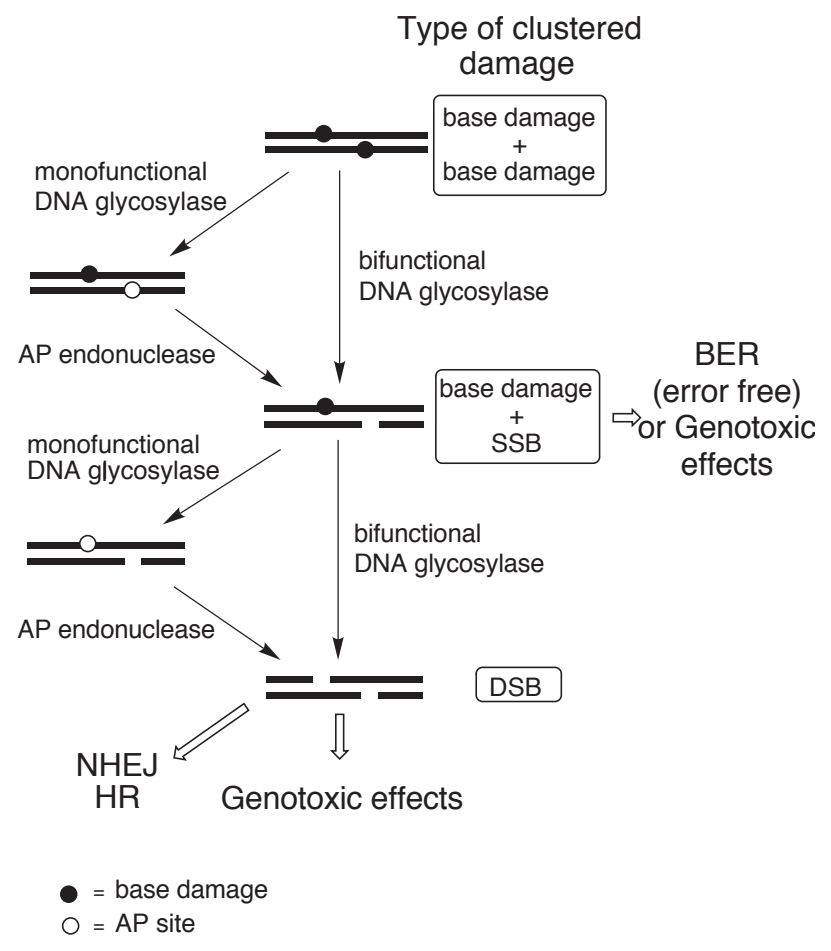

Fig. 4. Processing of clustered base lesions by BER enzymes. and one to three base pairs away is inefficiently removed from DNA by Endo III, whereas an AP site in similar arrangements was readily processed. This suggests that the glycosylase activity of Endo III for DHT is strongly inhibited by the cluster's structure, but that the AP lyase activity is not. These results suggest a novel mechanism for DSB production from a latent type of clustered DNA damage which is instigated by repair. Harison et al. (1998) showed that the activities of Endo III and Endo VIII, which share repair activity for oxidative pyrimidines in E. coli, are not affected by opposing gap damage sites which are spaced apart by a span of over 3 nucleotides. For clustered base lesions, Fpg exhibits a spatial dependence similar to that of Endo III and Endo VIII (Harison et al., 1999). These results suggest that SSBs on opposing strands (or a gap) within two base pairs away are strongly inhibitory to DNA glycosylases and prevent the generation of DSBs. APE1, a major human AP endonuclease, generates DSBs from clustered DNA damage containing an AP site and a SSB (DavidCordonnier et al., 2002). Clustered DNA damage containing two base lesions shows some differences from that containing a SSB. The excision activity of Endo III for DHT varies with neighboring damage, and DHT opposing an AP site is not readily converted to a DSB (David-Cordonnier et al., 2000). Although DHT by itself is not genotoxic, similar types of clustered DNA damage containing a genotoxic lesion might remain in place for a long time if appropriate repair does not take place, and these lesions could eventually lead to lethal or mutagenic events in vivo. The efficiencies of various DNA glycosylases in the repair of clustered DNA damage have been summarized by Blaisdell et al. (2001). However, other enzymes involved in DNA scission ends and in cross-link repair have not been studied.

The spatial distribution of clustered DNA damage in chromatin is also important in determining the biological consequences of clustered DNA damage. In V79 cells, $\mathrm{X}$-rays and $\alpha$-particles generate DNA fragments with distinct sizes which vary with an increase in dose (Newman et al., 1997). Thus, the fragment sizes resulting from direct or indirect (repair enzyme-catalyzed) DSBs may reflect both the track structure of the incident IR and the chromatin structure of the cells. The loss of a chromosome fragment bracketed by two DSBs results in a deletion, and the size of these IR-induced deletions is revealed by the fragment sizes seen after particular IR doses.

There are a number of other factors involved in producing the biological consequences of clustered DNA damage. One of these is the genetic background of the cells. Weber and Flentje (1993) reported wide variations of DSB yields among different cell types. The yield of DSBs measured with the FAR assay in chinese hamster lung fibroblast V79 cells was twice as high as that seen in established human tumor cells. 


\section{Perspectives}

A number of studies of clustered DNA damage suggest that clustered DNA damage is a key factor in the biological consequences of IR. Recent data also lends support for the existence of a crucial role for clustered DNA damage in cellular effects of IR (Ali et al., 2004). High LET radiations such as HZE particles are believed to lead to severe biological consequences due to the formation of clustered DNA damage. However, experimental studies show contradictory results when considering the question of whether the yield of clustered DNA damage is higher for high LET radiation than for low LET radiation. It is necessary to accurately measure the yield of clustered DNA damage in vitro and in vivo, and also to define the chemical nature of the clustered DNA damage actually formed in living cells after exposure to IR. Knowledge of DNA repair mechanisms has advanced tremendously during the last two decades, and thus various DNA repair enzymes are now available to detect base lesions in clustered DNA damage, and can also be used to characterize the nature of clustered DNA damage. The role of sugars and of cross-link lesions in clustered DNA damage needs to be assessed in future studies. Assessment of the number and spatial distributions of individual lesions in clustered DNA damage is also important. If these factors can be carefully described and characterized, it will be possible to build reasonable and realistic in vitro models of clustered DNA damage which can serve as a bridge between in vitro and in vivo studies of radiation biology and chemistry.

\section{References}

Ahnstrom, G. and Erixon, K. (1973) Radiation induced strand breakage in DNA from mammalian cells. Strand separation in alkaline solution. Int. J. Radiat. Biol., 23, 285-289.

Ali, M.M., Kurisu, S., Yoshioka, Y., Terato, H., Ohyama, Y., Kubo, K. and Ide, H. (2004) Detection of endonuclease IIIand 8-oxoguanine glycosylase-sensitive base modifications in $\gamma$-irradiated DNA and cells by the aldehyde reactive probe (ARP) assay. J. Radiat. Res., 45, 229-237.

Blaisdell, J.O., Harrison, L. and Wallace, S.S. (2001) Base excision repair processing of radiation-induced clustered DNA lesions. Radiat. Protect. Dosimet., 97, 25-31.

Blaisdell, J.O. and Wallace, S.S. (2001) Abortive baseexcision repair of radiation-induced clustered DNA lesions in Escherichia coli. Proc. Natl. Acad. Sci. U.S.A., 98, 7426-7430.

Blocher, D. (1990) In CHEF electrophoresis a linear induction of DSB corresponds to a nonlinear fraction of extracted DNA with dose. Int. J. Radiat. Biol., 57, 7-12.

Blocher, D. and Kuhni, M. (1990) DNA double-strand break analysis by CHEF electrophoresis. Int. J. Radiat. Biol., 58, 23-34.

Blocher, D., Sigut, D. and Hannan, M.A. (1991) Fibroblasts form ataxia telangiectasia (AT) and AT heterozygotes show an enhanced level of residual DNA double-strand breaks after low dose-rate $\gamma$-irradiation as assayed by pulsed field gel electrophoresis. Int. J. Radiat. Biol., 60, 791-802.
Bocker, W., Bauch, T., Muller, W.-U. and Streffer, C. (1997) Image analysis of comet assay measurement. Int. J. Radiat. Biol., 72, 449-460.

Bradley, M.O. and Kohn, K.W. (1979) X-ray induced DNA double strand breaks production and repair in mammalian cells as measured by neutral filter elution. Nucleic Acids Res., 7, 793-804.

Bryant, P.E. and Blocher, D. (1980) Measurement of the kinetics of DNA double strand break repair in Ehrich ascites tumour cells using the unwinding method. Int. J. Radiat. Biol., 38, 335-347.

Butts, J.J. and Katz, R. (1967) Theory of RBE for heavy ion bombardment of dry enzyme and viruses. Radiat. Res., 30 , 855-871.

Carle, G.F. and Olsen, M.V. (1984) Separation of chromosomal DNA molecules from yeast by orthogonal-field-alternation gel electrophoresis of DNA. 3. Effect of electrical field shape. Biochemistry, 27, 9216-9221.

Chaundhry, M.A. and Weinfeld, M. (1995) The action of Escherichia coli endonuclease III on multiply damaged sites in DNA. J. Mol. Biol., 249, 914-922.

Christensen, R.C., Tobias, C.A. and Taylor, W.D. (1972) Heavy-ion-induced single- and double-strand breaks in phiX-174 replicative form DNA. Int. J. Radiat. Biol., 22, 457-477.

David-Cordonnier, M., Cunniffe, S.M.T., Hickson, I.D. and O'Neill, P. (2002) Efficiency of incision of an AP site within clustered DNA damage by the major human AP endonuclease. Biochemistry, 41, 634-642.

David-Cordonnier, M., Laval, J. and O'Neill, P. (2000) Clustered DNA damage, influence on damage excision by XRS5 nuclear extracts and Escherichia coli Nth and Fpg proteins. J. Biol. Chem., 275, 11865-11873.

Evans, M.D., Podmore, J.D., Daly, G.J., Perrett, D., Lunec, J. and Herbert, K.E. (1995) Detection of purine lesions in cellular DNA using single cell gel electrophoresis with Fpg protein. Biochem. Soc. Transact., 23, 434S.

Filippova, E.M., Monteleone, D.C., Trunk, J.G., Sutherland, B.M., Quake, S.R. and Sutherland, J.C. (2003) Quantifying double-strand breaks and clustered DNA damages in DNA by single-molecule laser fluorescence sizing. Biophys. J., 84, 1281-1290.

Foray, N., Fertil, B., Alsbeth, M.G.A., Badie, C., Chavaudra, N., Iliakis, G. and Malaise, E.P. (1996) Dose-rate effect on radiation-induced DNA double strand breaks in the human fibroblast HF19 cell line. Int. J. Radiat. Biol., 69, 241-249.

Freeman, S.E., Blackett, A.D., Monteleone, D.C., Setlow, R.B., Sutherland, B.M. and Sutherland, J.C. (1986) Quantitation of radiation-, chemical-, or enzyme-induced single strand breaks in nonradioactive DNA by alkaline gel electrophoresis: application to pyrimidine dimers. Anal. Biochem., 158, 119-129.

Friedl, A.A., Beisker, W., Hahn, K., Eckardt-Schupp, F. and Kellerer, A.M. (1993) Application of pulse field gel electrophoresis to determine $\gamma$-ray-induced double-strand breaks in yeast chromosomal molecules. Int. J. Radiat. Res., 63, 173-181

Georgakilas, A.G., Bennet, P.V. and Sutheland, B.M. (2002) High efficiency detection of bistranded abasic clusters in 
$\gamma$-irradiated DNA by putrescine. Nucleic Acids Res., 30, 2800-2808.

Goodhead, D.T. (1994) Initial events in the cellular effects of ionizing radiations: clustered damage in DNA. Int. J. Radiat. Biol., 65, 7-17.

Gulston, M., Fulford, J. Jenner, T., de Lara, C. and O'Neill, P. (2002) Clustered DNA damage induced by $\gamma$ radiation in human fibroblast (HF19), hamster (V79-4) cells and plasmid DNA is revealed as Fpg and Nth sensitive sites. Nucleic Acids Res., 30, 3464-3472.

Hagen, U. (1974) Ultracentrifugation. In Experimental methods in biophysical chemistry (Nicolau, C. ed.) Wiley, New York, pp. 164-206.

Hagen, U. (1989) Radiation biology in space: a critical review. Adv. Space Res., 9, 3-8.

Harrison, L., Hatahet, Z., Purmal, A.A. and Wallace, S.S. (1998) Multiply damaged sites in DNA: interactions with Escherichia coli endonuclease III and VIII. Nucleic Acids Res., 26, 932-941.

Hall, D.B., Holmlin, R.E. and Barton, J.K. (1996) Oxidative DNA damage through long-range electron transfer. Nature, 382, 731-735.

Harrison, L., Hatahet, Z. and Wallace, S.S. (1999) In vitro repair of synthetic ionizing radiation-induced damaged DNA sites. J. Mol. Biol., 290, 667-684.

Hoeijmakers, J. H. (2001) Genome maintenance mechanisms for preventing cancer. Nature, 411, 366-374.

ICRU Report 16 (1970) Linear energy transfer. International Commission on Radiation Units and Measurements, Washington.

Jones, G.D.D., Milligan J.R., Ward, J.F., Calabro-Jones, P.M. and Aguilera. J.A. (1993) Yield of strand breaks as a function of scavenger concentration and LET for SV40 irradiated with ${ }^{4} \mathrm{He}$ ions. Radiat. Res., 136, 190-196.

Jostes, R.F., Hui, T.E. and Cross, F.T. (1993) Single-cell gel technique supports hit probability calculation. Health Phys., 64, 675-679.

Kohn, K.W., Erickson, L.C., Ewig, R.A.G. and Friedman, C.A. (1976) Fractionation of DNA from mammalian cells by alkaline elution. Biochemistry, 15, 4629-4637.

McEwen, C.R. (1967) Tables for estimating sedimentation through linear concentration gradients of sucrose solution. Anal. Biochem., 20, 114-149.

Michalik, V. (1992) Model of DNA damage induced by radiations of various quality. Int. J. Radiat. Res., 62, 9-20.

Milligan, J.R., Aguilera, J.A., Wu, C.C.L., Ng, J.Y.-Y. and Ward, J.F. (1996) The difference that linear energy transfer makes to precursors of DNA strand breaks. Radiat. Res., 145, 442-448.

Milligan, J.R., Aguilera, J.A., Nguyen, T.-T.D., Paglinawan, R.A. and Ward, J.F. (2000) DNA strand-break yields after post-irradiation incubation with base excision repair endonucleases implicate hydroxyl radical pairs in doublestrand break formation. Int. J. Radiat. Res., 76, 1475-1483.

Newman, H.C., Prise, K.M., Folkard, M. and Michael, B.D. (1997) DNA double-strand break distributions in X-ray and $\alpha$-particle irradiated V79 cells: evidence for non-random breakage. Int. J. Radiat. Biol., 71, 347-363.

Nikjoo, H., Uehara, S., Wilson, W.E., Hoshi, M. and Goodhead, D.T. (1998) Track structure in radiation biology: theory and applications. Int. J. Radiat. Biol., 73, 355-364.

Olive, P.L. (1999) DNA damage and repair in individual cells: applications of the comet assay in radiobiology. Int. J. Radiat. Biol., 75, 395-405.

Olive, P.L. and Banath, J.P. (1993) Detection of DNA doublestrand breaks through the cell cycle after exposure to X-rays, bleomycin, etoposide and ${ }^{125} \mathrm{IdU}$ rd. Int. J. Radiat. Biol., 64, 349-358.

Ostling, O. and Johansen, J.K. (1984) Microelectrophoretic study of radiation-induced DNA damage in individual mammalian cells. Biochem. Biophys. Res. Commun., 123, 291-298.

Prise, K.M., Pullar, C.H.L. and Michael, B.D. (1999) A study of endonuclease III-sensitive sites in irradiated DNA: detection of $\alpha$-particle-induced oxidative damage. Carcinogenesis, 20, 905-909.

Roots, R., Holley, W., Chatterjee, A., Irizarry, M. and Kraft, G. (1990) The formation of strand breaks in DNA after highLET irradiation: a comparison of data from in vitro and cellular systems. Int. J. Radiat. Biol., 58, 55-69.

Roots, R. and Okada, S. (1975) Estimation of life times and diffusion distances of radicals involved in X-ray-induced DNA strand breaks or killing of mammalian cells. Radiat. Res., 64, 306-320.

Rydberg, B. (1975) The rate of strand separation in alkali of DNA of irradiated mammalian cells. Radiat. Res., 61 , 274-287.

Rydberg, B. (1996) Clusters of DNA damage induced by ionizing radiation: Formation of short DNA fragments. II. Experimental detection. Radiat. Res., 145, 200-209.

Schwartz, D.C. and Cantor, C.R. (1984) Separation of yeast chromosome-sized DNAs by pulse field gel electrophoresis. Cell, 37, 67-75.

Simpson, J.A. (1983) Elemental and isotopic composition of the galactic cosmic rays. Ann. Rev. Nucleic Part. Sci., 33, 323-381.

Sutherland, B.M., Bennet, P.V., Cintron-Torres, N., Hada, M., Trunk, J., Monteleone, D., Sutherland, J.C., Laval, J., Stanislaus, M. and Gewirtz, A. (2002) Clustered DNA damages induced in human hematopoietic cells by low doses of ionizing radiation. J. Radiat. Res., 43, S149-S152.

Sutherland, B.M., Bennet, P.V. and Sutherland, J.C. (1996) Double strand breaks induced by low doses of $\gamma$ rays or heavy ions: quantitation in nonradioactive human DNA. Anal. Biochem., 239, 53-60.

Sutherland, B.M., Bennet, P.V., Sidorkina, O. and Laval, J. (2000) Clustered DNA damages induced in isolated DNA and in human cells by low doses of ionizing radiation. Proc. Natl. Acad. Sci. U.S.A., 97, 103-108.

Sutherland, B.M., Georgakilas, A.G., Bennet, P.V., Laval, J. and Sutherland, J.C. (2003) Quantifying clustered DNA damage induction and repair by gel electrophoresis, electronic imaging and number average length analysis. Mutat. Res., 
531, 93-107.

Taucher-Sholz, G. and Kraft, G. (1999) Influence of radiation quality on the yield of DNA strand breaks in SV40 DNA irradiated in solution. Radiat. Res., 161, 595-604.

Van Touw, J.H., Verberne, J.B., Retel, J. and Loman, H. (1985) Radiation-induced strand breaks in $\phi \mathrm{X}-174$ replicative form DNA: an improved experimental and theoretical approach. Int. J. Radiat. Biol., 48, 567-578.

von Sonntag, C. (1987) The chemical basis of radiation biology. Taylor \& Francis, London, UK.

Wallace, S.S. (1998) Enzymatic processing of radiation-induced free radical damage in DNA. Radiat. Res., 150, S60-S79.

Ward, J.F. (1981) Some biochemical consequences of the spatial distribution of ionizing radiation-produced free radicals. Radiat. Res., 86, 185-195.

Ward, J.F. (1988) DNA damage produced by ionizing radiation in mammalian cells: identities, mechanisms of formation, and reparability. Prog. Nucleic Acid Res., 35, 95-125.

Ward, J.F. (1994) The complexity of DNA damage: relevance to biological consequences. Int. J. Radiat. Biol., 66, 427-432.

Weber, K.J. and Flentje, M. (1993) Lethality of heavy ioninduced DNA double-strand breaks in mammalian cells. Int. J. Radiat. Biol., 64, 169-178.

Wood, R.D., Mitchell, M., Sgouros, J. and Lindahl, T. (2001) Human DNA repair genes. Science, 291, 1284-1289.

Woods, W. (1981) Quantification of the repair of gammaradiation-induced double-strand DNA breaks in human fibroblasts. Biochim. Biophys. Acta, 655, 342-348.

Yang, N., Galick, H. and Wallace, S.S. (2004) Attempted base excision repair of ionizing radiation damage in human lymphoblastoid cells produces lethal and mutagenic double strand breaks. DNA Repair, 3, 1323-1334. 